\title{
Analysis of methods for CubeSat mission design based on in-orbit results of KRAKsat mission
}

\author{
${ }^{1}$ Alicja Musiał, ${ }^{2}$ Dominik Markowski, ${ }^{3}$ Jan Życzkowski, ${ }^{4}$ Krzysztof A. Cyran \\ ${ }^{1}$ Jagiellonian University, Kraków, Poland. She is now with KP Labs, Gliwice, Poland. \\ ${ }^{2,3}$ AGH University of Science and Technology, Faculty of Electrical Engineering, Automatics, Computer \\ Science and Biomedical Engineering, Kraków, Poland. \\ ${ }^{4}$ Virtual Flight Laboratory, Faculty of Automatic Control, Electronics and Computer Science, Silesian University \\ of Technology, Gliwice, Poland.
}

Received: August 10, 2021. Revised: September 13, 2021. Accepted: September 17, 2021.

Published: September 21, 2021.

\begin{abstract}
The success rate of currently performed CubeSat missions shows that despite their popularity, small satellites are still not as reliable as larger platforms. This research was conducted to analyse in-orbit experience from the KRAKsat mission and discuss methods for mission design and engineering that can increase CubeSats reliability and prevent their failures. The main purpose was to define best practices and rules that should be followed during mission development and operations to ensure its success based on the overview of the lessons learned from KRAKsat and problems encountered during its mission. This paper summarizes the experiences obtained and provides methods that can be used while carrying out future robust CubeSat projects. It was written to prove that there are some parts of the small satellite missions that are often neglected in the university-led projects and, by ensuring proper testing and planning before the actual mission, its reliability can increase. The following analysis could be used as a guide during the development of the next CubeSat projects.
\end{abstract}

Keywords-CubeSat, satellite mission design, system engineering, KRAKsat, nanosatellite, satellite communication.

\section{INTRODUCTION}

$I^{N}$ $\mathrm{N}$ recent years the market for small satellites has accelerated rapidly. CubeSat standard, which was introduced in 1999 by California Polytechnic State University and Stanford University [1], became widely used as a platform for both commercial and educational purposes. The term CubeSat is used to describe a small satellite that consists of a multiplication of basic unit $1 \mathrm{U}(10 \times 10 \times 10 \mathrm{~cm}$ cubes $)$. The device's design has to be compliant with standards defined in the CubeSat Design Specification [2] and follow the requirements to ensure safety rules fulfilment and correspondence with the deployment mechanisms.

CubeSat standard, given its simplicity, affordability, and short development time (on average less than two years [3]), quickly gained attention and became popular in the academic environment as a tool for education and new technologies development. The platform has proven itself to be comparative in terms of research capabilities, reducing the threshold for entry into space technology studies [4-6]. According to the Nanosats Database, around 30\% of all 1553 CubeSats launched were university-led projects and their number is continuously growing [7].

The significant interest in the platform, however, did not come with the reliability - robustness statistics show that in years 2005-2018 only around $60 \%$ of CubeSats fully or partially succeeded with their missions [8]. Whereas infant mortality rate, defined as a failure just after getting into the space environment, has decreased remarkably (at the beginning of the decade more than a half of small satellite projects did not even finish the commissioning phase - now the number is around 20\%), CubeSats are still in the process of developing and proving their reliability.

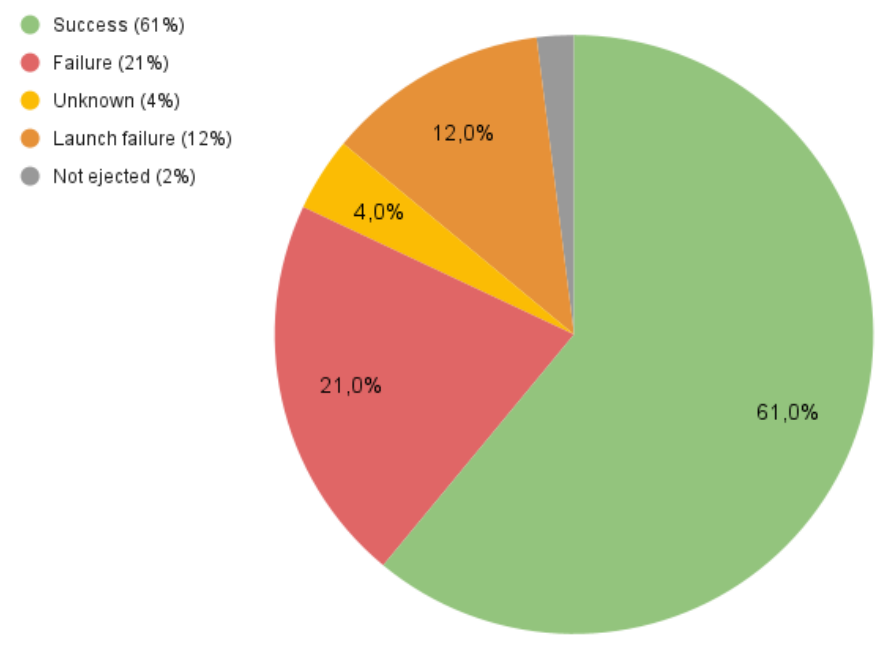

Fig. 1 CubeSat's status in years 2005-2018 (based on [8]) 
There are many reasons for the failures of student-driven nanosatellite projects, depending on the mission goals and details of the specific implementations and chosen components. Short deadlines, limited resources ([3]), tests shortages ([9]), and lack of experience significantly impact mission failure rate. To improve the likelihood of achieving the small mission success, every development team should benefit from experience from similar previous missions and analyse existing lessons learned to avoid mistakes that were already committed.

Currently available papers on the CubeSat reliability topic can be generally divided into two main categories: overall statistics of the total successes and failures of the small satellites' missions that analyse the whole industry robustness ([2], [3], [5], [7], [8]) and lessons learned from specific CubeSat projects ([9], [10], [22], [23], [24]). Both are remarkably valuable in the process of satellite development, as they emphasize different aspects of this kind of mission and give multiple points of view to the mission itself. There is, however, a common conclusion shared by practically all of them - that there are certain parts of the mission preparation and execution that are usually neglected and deficiencies in these areas could lead to a catastrophe. This paper is meant to be complemental to the existing literature in this research area from the perspective of the KRAKsat nanosatellite project. The study is then limited to this specific mission insight, as it contains experience gained during its operation only. Despite this limitation, it should be helpful for future researchers in the student satellite projects, as it includes unique observations and practical hints based on the problems the team had been forced to confront.

The following paragraphs focus on methods of CubeSat engineering and reliable mission design, based on KRAKsat in-orbit experience [10]. Analysis of lessons learned from the KRAKsat mission allows drawing conclusions that can serve as a base for forthcoming CubeSat university-led projects, presenting tools and best practices of engineering that can help with new missions' organization and development.

\section{MISSION DESIGN}

\section{A. Overview}

KRAKsat project was conducted by students from AGH University of Science and Technology and Jagiellonian University in Cracow. The main goal of the nanosatellite mission was to test an innovative attitude actuator for nanosatellites based on a ferromagnetic liquid - the Ferrofluid Reaction Wheel (FRW) [11]. FRW is a response to the needs of small satellites' platforms, where it is crucial to have precise control over the orientation in space, e.g., to be able to collect the energy from sunlight, communicate with ground station or point exactly at the specified target with a camera or a sensor.

The detailed description of the whole project and the mission design can be found in the KRAKsat documentation ([11]).

\section{B. Payload}

Attitude Determination and Control System (ADCS), which is responsible for orientation manoeuvres and pointing accuracy, usually relies on reaction wheels to execute the planned rotation of the spacecraft. The FRW was meant to become a competitive solution for reaction wheels used commonly in nanosatellite missions, due to its reliability, simplicity, and low-cost production. It is a device invented, designed, and manufactured by team members as a prototype of the reaction wheel based on 12 millilitres of ferrofluid as a rotating element. The whole idea is innovative, but not the first in the industry - the earliest fluid reaction wheel was successfully tested by the TechnoSat mission launched in 2017 [12]. Although the experiment utilized liquid metal and electromagnetic pump instead of ferrofluid, it showed that similar projects can become a low-budget substitute for traditional reaction wheels. Fully mounted FRW, ready for integrating with the satellite, is presented in Fig. 2.

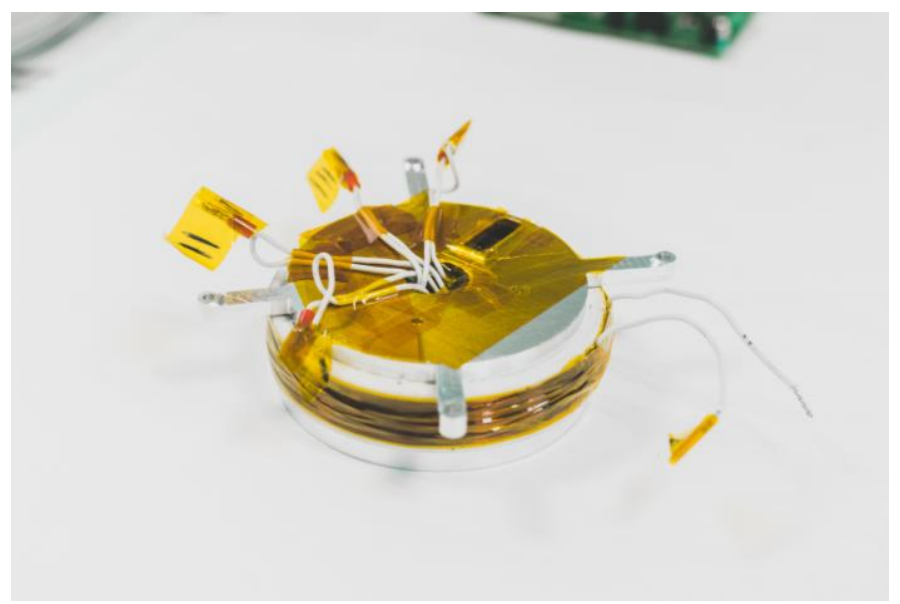

Fig. 2 Ferrofluid Reaction Wheel

FRW construction layout is presented in Fig. 3. Eight electromagnets are arranged around a torus-shaped container filled with ferrofluid. The container is made of stainless steel 316L. It is composed of two separate parts, which are later connected using laser welding. More information about the manufacturing process of the container can be found in reference [13]. To drain the heat away, FRW is covered with thermal paste and placed inside an aluminium wheel case. Each electromagnet is connected in series with the one on the opposing side. For more detailed information about FRW construction, see [11]. 


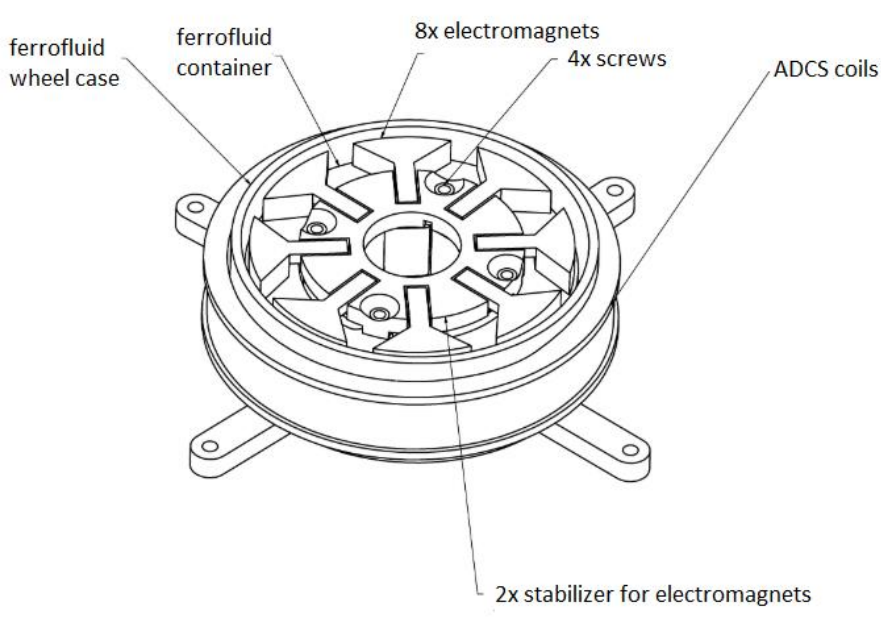

Fig. 3 FRW construction layout

To create torque, that can affect the satellite orientation, ferrofluid is accelerated in the torus by switching electromagnets on and off in a specific order. To improve FRW performance different strategies have been proposed including half step, full step, steering with closed-loop, and with PID regulator. To test the real performance of the FRW an IMU sensor had been placed inside the device. Measurements from the magnetometer and gyroscope can be then combined to calculate the rotation of the whole device. Due to the principle of conservation of momentum, the torque created by FRW and ferrofluid velocity can also be obtained.

The idea of FRW operation has been tested on a larger prototype [14, 15] and using physics simulation software [16]. Both actions confirmed the correctness of the approach taken and led to the construction of the FRW in size appropriate for a nanosatellite.

To verify the possible performance and measure torque created by the FRW, a ground test device has been constructed. It consisted of the FRW hanging on a fishing line. After turning the payload on, a change in the angular speed was measured which allowed calculating a generated torque. Based on the examination results, the minimal torque measured was around $9.4 * 10^{-7} \mathrm{Nm}$. However, in orbit, according to the simulation, it can be much larger, due to the neglection of forces related to viscosity and the microgravity environment. The whole experiment is described in detail in the reference [16].

After the thermo-vacuum chamber, random vibration tests, tightness tests, and electromagnetic screening, which proved the FRW meets the CubeSat payload's requirements, it was placed inside the KRAKsat satellite. Although the satellite was launched to orbit successfully, the performance of the FRW in microgravity is still unknown. The experiment had been carried out, but the problems with flash memory and power system, described in the following chapters, made downloading of the results impossible.

\section{Satellite bus}

It is considered a normal practice to use the off-the-shelf universal satellite bus as a platform for the university-led experimental payload that implements specific mission objectives $[9,22]$. The same approach is used in the KRAKsat mission, in which the commercial SR-NANO-BUS is utilized. It contains of four distinguished subsystems: On-Board Computer (OBC) with ADCS included, Electrical Power System (EPS), Communication Module (COMM), and $1 \mathrm{U}$ standardized CubeSat frame. These components provide all functionalities necessary for a satellite to operate on LEO.

The OBC is responsible for command and data handling between all subsystems. Its software, which was a part of the platform, implements most of the procedures and controls the work of all components and the mission lifecycle. A memory located on the $\mathrm{OBC}$ board (8 MB flash) stores satellite's telemetry data and logs produced by the payload module. OBC board contains also the ADCS microcontroller that provides backup detumbling algorithms that can be used during the mission instead of experimental FRW.

The KRAKsat's EPS (also a part of the platform) controls and monitors battery voltage and the satellite's power consumption. This module should be capable of disabling specific units if the voltage level is too low and enabling them when solar panels recharge the battery above a defined threshold. It consists of two boards that allow $3200 \mathrm{mAh}$ batteries charging and provide $3.3 \mathrm{~V}$ and $12 \mathrm{~V}$ to all satellite subsystems.

The COMM consists of two redundant UHF transceivers. It operates in the amateur's frequency band $430-440 \mathrm{MHz}$ with the transmission data rate of $9600 \mathrm{~b} / \mathrm{s}$ uplink (GFSK modulation) and $1200 \mathrm{~b} / \mathrm{s}$ downlink (AFSK modulation). It is responsible for communication with ground stations to either update satellite settings, schedule tasks or transmit telemetry and $\operatorname{logs}$ to the ground station.

The $1 \mathrm{U}$ frame is a mechanical structure that connects all the components and implements a passive heat regulation. Its mechanical interface interacts with the launch system - that is why it has to be designed and tested according to the CubeSat Design Specification [2]. The whole utilized platform, SRNANO-BUS, is described in detail in the KRAKsat documentation [11].

\section{IN-ORBIT EXPERIENCE}

In January 2019 KRAKsat has been finally integrated, with cooperation with platform provider, and, a few months later, in April, launched on board of Cygnus NG-11 on Antares rocket with delivery to the International Space Station (ISS). The actual mission began with deployment from the ISS via NanoRacks deployer on the 3rd of July 2019, 11:50 UTC. After awaiting the mandatory safety delay period of 30 minutes, the EPS module should have turned on the power to all satellite subsystems, starting the antennas deployment and periodic beacon messages transmission. A dedicated ground station located in Poland together with a word-wide amateur radio network SatNOGS had been used to listen for the expected incoming signals since the moment of the deployment, but no signal was received at first - there was no communication established and no downlink received until the $16^{\text {th }}$ of July. It was assumed, based on the later observations of 
battery voltage and satellite internal timestamp value, that the most probable explanation of the radio silence was a temporary power switch malfunction, which could have caused a delay in the satellite's activation procedure.

The first beacon radio signal was received by the SatNOGS station and the main station in Warsaw, Poland on the 16th of July 2019. KRAKsat turned itself on with almost full battery voltage and after obtaining the successful two-way communication, the proper mission began.

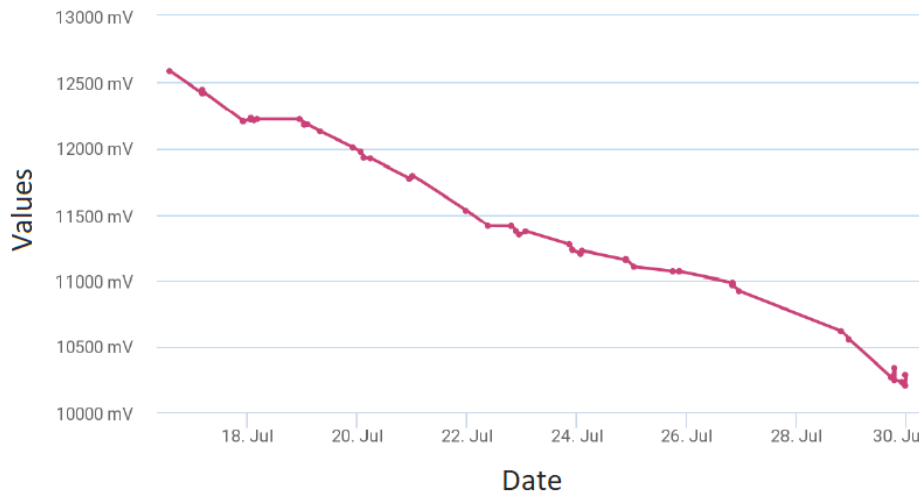

Fig. 4 KRAKsat satellite's battery voltage (from reference [10])

In the period between the 16th to the 30th of July the team attempted to fulfil mission objectives by turning on the FRW experiment. Unfortunately, the main goal could not be reached due to a number of errors that are described in detail in reference [10]. Because of the negative energy budget (Fig. 4), lack of hysteresis and EPS problems, two weeks from the first signal, on the 30th of July, the satellite became nonoperational, stuck in a reboot loop. Since then KRAKsat constantly discharges completely, shutting down all the subsystems, which immediately causes a small power increase and a restart. The restart is followed by a beacon frame transmission, which again discharges the battery and causes a satellite's reboot. Despite numerous attempts to amend such behaviour, the satellite is not responsive and incapable to charge to the operational level. The frequency of the reboots is fluctuating, depending mostly on the current level of illumination in orbit (Fig. 5 and Fig. 6).

The comprehensive description of the KRAKsat mission's phases can be found in the reference [10].

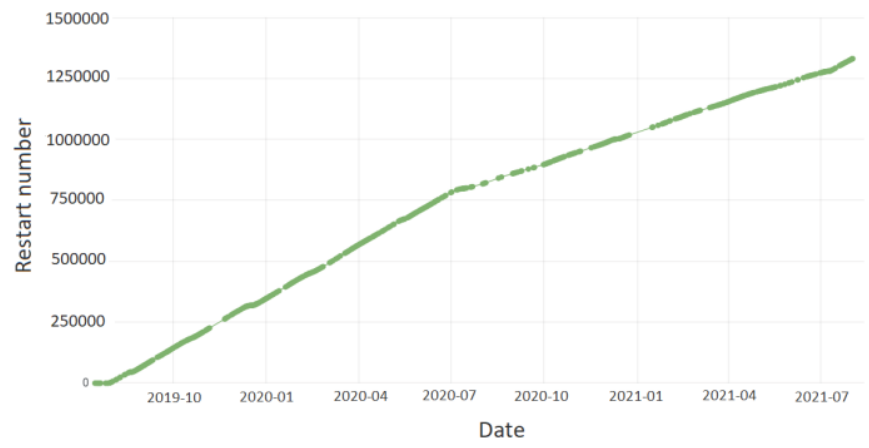

Fig. 5 Number of KRAKsat satellite's restarts in the mission time (July 2019 - August 2021)

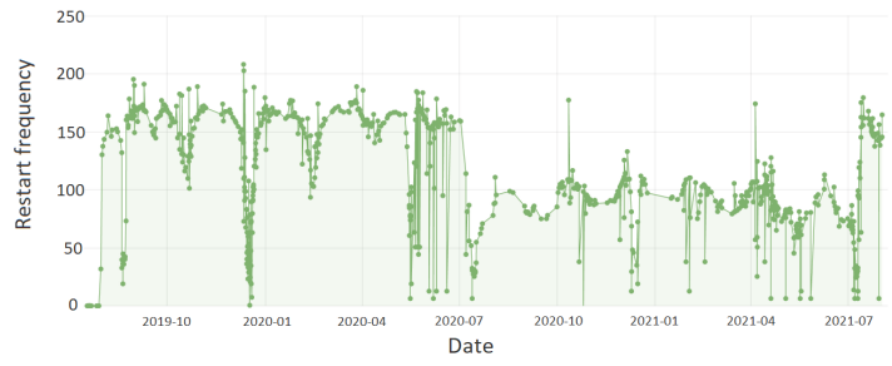

Fig. 6 Change of KRAKsat satellite's reboots frequency in one orbit in the mission time (July 2019 - August 2021)

\section{METHODS FOR CUBESAT MISSION DESIGN}

The subsequent sections present methods and best practices for CubeSat mission design. All presented principles and inferences are based on lessons learned from KRAKsat failure. The chapter is divided into several modules that highlight the most important parts of the small satellite mission connected to the KRAKsat in-orbit issues. Problems are described in detail together with proposed solutions to emphasise which best practices can play an important role in the satellite mission development.

\section{A. Electrical Power System}

Regardless of the size of the satellite, power management and the design of the Electrical Power System (EPS) is always a critical issue during mission development. However, smaller CubeSats require an even more reasonable distribution of energy for all the subsystems than bigger satellites and very precise energy budget calculations. There has to be a possibility to control the power draw at any point of the mission, to avoid the complete discharge of the battery [17]. It was not implemented in KRAKsat and its lack had a significant impact on the mission [10]. As the energy budget was not determined properly, the voltage level of the satellite's batteries has been constantly decreasing since the mission start - until it fell into a reboot loop.

State-of-the-art technology for CubeSat power system management is usually based on the high-effective solar cells and lithium-ion batteries [1], mostly because of their convenient size and low cost. Components' properties should be the base for the precise energy budget calculation. Orbit Average Power (OAP), which determines how much power is available per orbit, depends on the characteristics of solar panels' - position, quantity, efficiency on the different angles relative to the sun (depending on the chosen orbit), and the possible orientation of the entire CubeSat [17]. According to reference [18], the efficiency of power processing should also be taken into consideration - EPS subsystem consists not only of solar arrays and batteries but also of chargers, converters, and switches on which there may also be small energy losses. The satellite's power budget can be then calculated by summing up all modules' energy consumption (considering their duty cycle) which makes an Average Power Used (APU). After applying a wide power margin [19], the APU can be subtracted from OAP, resulting in a power budget that has to be a positive number. It should be also considered that the 
solar cells and batteries will slowly degrade in the space environment, so their effectiveness will decline over time.

During KRAKsat development, the energy budget was calculated imprecisely, and it was based only on the theoretical evaluations instead of the real measurements. Lack of proper computation caused the unexpected deficit that quickly led to the failure of the mission. Additionally, there was no hysteresis implemented, so once the battery has discharged, KRAKsat became stuck in a reboot loop until the moment of writing (with 1,332,941 reboots on the counter [20]). As is highlighted in [17] and [19], the EPS should control the restart until the battery is recharged to a reasonable level to avoid similar problems.

All modules and payloads should have a possibility to be switched off individually - in the event of anomaly they could be temporarily turned off; the separation allows also to precisely monitor the power consumption of each one of them [17]. Measuring the current energy usage can also help determine the source of the failure in case of potential power problems. There was no emergency option to shut down satellite subsystems permanently and turn off their power in KRAKsat - all the modules were activated immediately after a reboot, consuming all of the energy surpluses. The safety mechanisms were therefore not implemented, which was indisputably one of the major causes of the mission failure.

\section{B. Software}

Satellite's software can be classified as the on-board computer software (responsible for general management of the spacecraft operations, housekeeping, and telemetry supervision), and as a payload controller software (experiment-related activities execution). In this section, we will focus on best practices concerning both.

According to [1], the software should preferably be based on a real-time operating system (RTOS), with complexity related to objectives and requirements of the specific mission. Independently from its convolutedness, the software update or patching should be available [25]. The presence of a properly designed bootloader and slot management system, that allows storing few images of the main program, often determines the mission's success in case of problems. The ability to replace any code during operation is a capability that can save a mission - even if the radio limits restrict the upload speed [24].

Another important feature of the satellite $\mathrm{OBC}$ software is logging. Every event that happened during the mission, should be saved in memory, with particular emphasis on errors. Such a design allows to easily recreate the timeline of the subnormal behaviour of the CubeSat and debug any problems encountered in-orbit. Data from the payload controller should also be included to be able to perform the main experiment and analyse its outcome.

To perform time-related and precise operations, accurate time measurement is needed. There are multiple hardware solutions for timer implementation along with software synchronization that can help to manage time during the mission. The scheduler should be implemented, which allows the operations team to plan operations in advance or periodically [5].
Recurring telemetry collection from all the sensors and subsystems requires its storing. For that purpose, a data pool can be created, defined as a container for all current telemetry values [25]. It is then easier to create a response for a telecommand demanding data from the specific sensor - we don't have to wait until it is sampled and calculated, getting it immediately from the data pool instead.

The implementation of a file system is also an important part of the RTOS. Without a file system operating a mission turns out to be quite a challenge. KRAKsat sensors' telemetry data was stored directly in low-level buffers and downloading it required a reference to the specific address in the memory. A rule of thumb should be to use already existing concepts and solutions to facilitate operations with a satellite already in orbit.

All software should be developed and tested while keeping in mind, how it is going to be used during the actual space mission. There is no need to make it too complex, as it can complicate already short communication sessions. Unit and component level tests should be used together with integration and operational tests [1]. Utilization of a version control system and CI that automatically builds the project and runs tests also should be a standard. Very limited software testing in the KRAKsat project together with lack of automatization led to multiple errors and bugs that were discovered in orbit, including a major cleaning flash problem that prevented us from downloading experiment data from the device.

In KRAKsat the software issues were very complex because of improper modes' design. The state machine automatically launched a cycle, including diagnostics and experiment, after every satellite's reboot, which resulted in enormous and unnecessary energy consumption after every restart. A reboot should be considered a part of normal operations that do not trigger any energy-consuming or time-consuming action. In order to prevent problems related to latch-ups, it is even recommended to schedule reboots every set amount of time (e.g. once a day) [24].

One of the good practices is to use an extensive mission parameters configuration. There should be no hard-coded values that prevent the operators' team to perform an action, even if it is the potentially dangerous one - double confirmation should be enough. A non-volatile place in the memory should be dedicated to configuration parameters, adjustable during the mission. There was no persistent state in KRAKsat that could allow the operations team e.g. to turn off satellite's subsystems permanently, so they don't start after a reboot. This kind of protection allows to get more energy when there is a problem with the energy budget, so it is worth implementing as a part of mentioned persistent settings.

It has not yet been established how significant effect software bugs have on a mission, but it should not be argued that the impact is high. The thing that prevents from acquiring knowledge about the exact percentage of failures related to software problems is their invisibility - they are hidden in the statistics of all satellites that stopped transmitting at some point in the mission for unknown reasons. Time spent on this subject is usually shorter than on the hardware or primary payload, which are the main concern of the development 
teams. A similar attitude was shared by the KRAKsat team, which was one of the reasons for this mission failure.

\section{Communication}

This paragraph will introduce methods for CubeSat communication system design from the perspective of mission operations. Together with the software, the communication topic is often ignored, and teams only begin to pay attention to the ground setup only in the final stages of the mission preparation. Based on the KRAKsat in-orbit experience [10], it can be reasonably assumed that taking care of the subject earlier could have a positive effect on the mission accomplishment.

To ensure proper communication with the CubeSat, two things should be guaranteed: reliable transmission and solid ground infrastructure. While planning the communication, proper modulation, baudrate, and frames protocol should be selected [5]. Depending on the chosen modulation, the signalto-noise ratio and bit error rates can have different values. To reduce them and achieve a reliable link, error correction needs to be used, which shortens the effective message length. Communication with KRAKsat was performed using AFSK modulation with baudrate $1200 \mathrm{~b} / \mathrm{s}$, which turned out to be inefficient, especially in the experiment data download. To provide efficient transfer of large amounts of data, the link budget should be computed and the communication properties should be chosen based on this calculation. KRAKsat data transmission used APRS protocol AX.25 with ASCII format. Therefore, the broadcast was time-consuming, causing energy waste. It also affected sensor data download - because of the incompatibility of binary data with the chosen protocol and a software defect, it was not possible to get telemetry properly.

The main functionality that allows diagnosing the satellite's mission constantly is a beacon. Its frequency should be chosen based on planned communication sessions' length and energy budget. As the typical session lasts around 10 minutes on LEO or SSO, intervals not longer than 7 minutes should be considered to get at least one beacon during every pass.

To fully exploit the mission's potential, different frequencies may be chosen for uplink and downlink [21]. It requires more complicated infrastructure in the ground station to handle UHF and VHF separately but ensures the most efficient usage of the radio link. The frequencies should be also selected considering neighbouring satellites' parameters. It would be best to avoid choosing the frequency that another device from the same launch is using - parallel communication with two satellites will cause interference between signals and the risk of transmission incomprehension will increase [10].

It is recommended to use more than one ground station to communicate with the satellite $[4,10]$. Lack of redundancy in the ground infrastructure can lead to massive problems in case of its malfunction. Multiple stations located in different localizations provide more frequent data download and protection in emergency situations. There are both commercial and non-commercial global networks (e.g. SatNOGS) available for usage, as well as possible agreements with other CubeSat operation teams on bilateral infrastructure sharing.
Using the amateur frequency allocation prevents CubeSat creators from downlink encryption - but uplink needs to be encrypted [1]. To prevent unauthorize access to the satellite and gaining control over it, commands should be secured using encryption algorithms (e.g. Advanced Encryption Standard, AES) or, at least, incoming packet counter incrementation.

\section{Mission analysis}

To ensure that the CubeSat mission fulfils its scientific goals, a mission analysis has to be made. Mission analysis is defined as a preparation for the actual satellite operation and identification of tasks that have to be performed during it [6]. It includes all activities related to recognition what are the main objectives of the mission and how to achieve them, as well as coordination of all requirements and their fulfilment evaluation. The superficial analysis of mission-related data and activities can lead to problems both with satellite communication sessions and project management in general. KRAKsat case shows how important the preparation and proper review of all the requirements is [10].

While working on the CubeSat project, the emphasis should be placed on amount of the telemetry that is going to be collected from available sensors and the radio link budget to guarantee that it will allow downloading data to the ground. Logging frequency, order of transmitted frames and the beacon's content should also be chosen deliberately to avoid a situation in which the needed data is unavailable for operators (as it happened to KRAKsat). Delivered platform and off-theshelf components should have their functionalities analysed and tested before the actual mission - to get to know their possibilities and limits in detail as early as possible. The already mentioned power budget should be precisely computed in versions for different possible scenarios, together with simulation of the satellite's time in the sunlight to compute power available to perform the actual experiment. Session length obtained from the orbit simulation should be carefully analysed to assure if there will be enough time to perform planned activities during communication time.

To achieve CubeSat experiment success, the mission analysis should be performed regularly during the development process, based on the incoming data from all evolving subsystems.

\section{E. Testing}

Testing is widely considered to be the key to the reliability and robustness of a CubeSat mission. Various approaches have been suggested to perform the most comprehensive on-ground testing of a satellite with usage of FlatSats, development boards, simulators, or electronical ground support equipment. A review of the literature on this subject, carried out for the needs of this paper, found that the most frequently used solutions include hardware-in-the-loop and software-in-theloop approaches $[19,23,26]$. In the case of the KRAKsat mission, no extensive system tests were conducted. Evaluation activities were limited to basic integration testing of payload controller software with on-board computer (OBC) simulator performed using custom, but simple FlatSat setup, that allowed 
to check the proper functioning of the payload. Incomplete testing and very delayed usage of real engineering models of OBC, EPS, and other subsystems led to many problems that were discovered during the actual mission.

Although two redundant UHF modules were placed inside the KRAKsat satellite, it was discovered during the mission, that radio's software implementation had some differences. As it was a part of a platform, the bug could have been discovered only in comprehensive whole-system tests. Testing could also prevent interfaces incompatibility, which is not an unpopular problem amongst CubeSats [10], [22]. In KRAKsat it led to an inability to download data from some sensors - data sent in binary format was incorrectly transmitted in ASCII without proper encoding.

As it is described in [24] having a full FlatSat setup with, at least, engineering models of all components, is preferable. Extensive flight software testing with simulators and hardwarein-the-loop setup assures that the performed evaluation will be adequate to the actual environment. By completing a wide variety of tests, the risk of mission failure can be significantly reduced.

\section{F. Project management and documentation}

In university-led teams working on CubeSats project management is often a neglected topic. Tense timelines and limited resources are a reason all attention is focused on the technical part of the project. This phenomenon causes multiple entries in the satellites' lessons learned highlighting the importance of proper project management and the significant impact of its lack on the success of the mission. Not only is the proper division of roles in the team important [22], but also proper task management and planning ahead. Before starting the actual mission, it is worth preparing an instruction for the operational team that will allow for the efficient run of the mission in various operational scenarios. Scenarios should include both success paths and actions in case of emergencies. There was no proper mission planning in KRAKsat and lack of strategy was one of the reasons for a delayed response to problems that occurred during operations [10]. For the same reason, detailed technical documentation of the project should be maintained. In case of failures, up-to-date and comprehensible description of all features becomes an invaluable tool for their effects' mitigation.

Although every satellite mission is different, it is essential to rely on previous teams' experience and knowledge. Some of the solutions that work in space are non-intuitive or difficult to verify before the flight - that is why consultation with experts should be the basis of the development of a new CubeSat. Many of the KRAKsat errors described in this paper could easily be avoided if the ideas were discussed with specialists before their implementation. Seeking advice from people experienced in the space industry and getting knowledge from already published papers or conference talks should serve as a foundation of every successful CubeSat project.

\section{CONCLUSIONS}

This paper has presented good practices and methods of CubeSat's mission design that are based on lessons learned from the KRAKsat satellite project. It is in fact supplemental to the previous research conducted in this field, as it confirms conclusions included in other lessons learned from the small satellite's projects ([9], [19], [22]) - that there are certain parts of the CubeSat engineering that is often neglected by the university-led teams. In general, results from our experience would seem to suggest that student groups tend not to focus on some of the aspects of the design - mostly testing, mission analysis, software, and project management - which, together with technical problems, can directly lead to mission loss. Despite all the CubeSat's advantages, it should be remembered that they are not damage-proof and the probability of failures is higher with small satellites than with larger ones, as there is no place for redundant units or space for additional shields that can protect the satellite from radiation. To mitigate the potential problems, it is extremely important to test all the solutions, components, and software before the flight, and plan the mission ahead to be ready for every possible scenario while in orbit.

We hope that our research will serve as a base for future studies of CubeSats engineering methods and workflow. The direction of the following research could be focused on enhancing the reliability of CubeSats so they provide more robust educational opportunities. It could be done e.g. by analysing current practices and introducing methods for better software engineering or ground segment infrastructure preparation that seem to be the weakest link in the small satellite industry. The future work could also be connected to the topic of the comprehensive testing of a CubeSat before the flight to achieve a bulletproof mission in-orbit.

\section{REFERENCES}

[1] State-of-the-Art. Small Spacecraft Technology, NASA, Small Spacecraft Systems Virtual Institute, October 2020, [Online]. Available: https://www.nasa.gov/sites/default/files/atoms/files/2020soa_final.pdf (accessed on $24^{\text {th }}$ July 2021)

[2] CubeSat Design Specification, Rev. 13, California Polytechnic State University, 2015

[3] A. Alanazi, J. Straub, Engineering Methodology for Student-Driven CubeSat in Aerospace, May 2019, DOI: 10.3390/aerospace6050054

[4] C. Nieto-Peroy, M. Reza Emami, CubeSat Mission: From Design To Operation in Applied Science, 2019, DOI: 10.3390/app9153110

[5] G. Sebastyen, S. Fujikawa, N. Galassi, A. Chuchra, Low Orbit Satellite Design, Space Technology Library, 2018

[6] P. Fortescue, G. Swinerd, J. Stark, Spacecraft systems engineering. Fourth Edition, A John Wiley \& Sons, Ltd., 2011

[7] Nanosats Database, [Online]. Available: https://www.nanosats.eu (accessed on $24^{\text {th }}$ July 2021)

[8] T. Vilella et al., Towards the Thousandth CubeSat: A Statistical Overview, International Journal of Aerospace Engineering, 2019, DOI: $10.1155 / 2019 / 5063145$

[9] M. Nowak, S. Lacour, A. Crouzier, L. David, V. Lapeyrere, G. Schworer, Short life and abrubt death of PicSat, a small $3 U$ CubeSat dreaming of exoplanet detection, January 2019, DOI: $10.1117 / 12.2313242$

[10] A. Kubera, D. Markowski, J. Życzkowski, KRAKsat Lessons Learned, Technical Report, December 2019, [Online]. Available: https://www.researchgate.net/publication/337874738_KRAKsat_Satellit 
e_Mission_-Lessons_Learned_EN (accessed on $24^{\text {th }}$ July 2021), DOI: $10.13140 /$ RG.2.2.14725.50408

[11] KRAKsat nanosatellite documentation, [Online]. Available: https://kraksat.gitbook.io/documentation/ (accessed on $24^{\text {th }}$ July 2021)

[12] TechnoSat, [Online]. Available: https://directory.eoportal.org/web/eoportal/satellite-missions/t/technosat (accessed on $24^{\text {th }}$ July 2021)

[13] R. Janiczak, Realization and examination of technology of welding a ferrofluid reservoir used in cosmonautics [in Polish], BoE Thesis, AGH University of Science and Technology in Kraków, 2019

[14] J. Życzkowski, Teoretical simulation of stabilization system for nanostelites based on the ferrofluid [in Polish], BoE Thesis, AGH University of Science and Technology in Kraków, 2018

[15] D. Markowski, Construction of the stabilization system for nanosatellite using ferrofluid [in Polish], BoE Thesis, AGH University of Science and Technology in Kraków, January 2017, DOI: 10.13140/RG.2.2.36320.71680

[16] J. Życzkowski, Ferrofluid reaction wheel for nanosatellites orientation control [in Polish], Master Thesis, AGH University of Science and Technology in Kraków, 2019

[17] C. Clark, R. Logan, Power Budgets for Mission Success, ClydeSpace, 28 April 2011, [Online]. Available: http://mstl.atl.calpoly.edu/ workshop/archive/2011/Spring/Day\%203/16 10\%20-\%20Clark\%20\%20Power\%20Budgets $\% 20$ for $\% 20$ CubeSat $\% 20$ Mission $\% 20$ Success.p df (accessed on $24^{\text {th }}$ July 2021)

[18] PW-Sat2 Electrical Power System. Preliminary Design Review, PWSat2, Students' Space Association, Warsaw University of Technology, [Online]. Available: https://pw-sat.pl/wp-content/uploads/2014/07/PWSat2-A-03.00-EPS-PRR-EN-v1.1.pdf (accessed on 25 th July 2021)

[19] S. Ruckerl et al., First flight results of the MOVE-II Satellite, 33rd Annual AIAA/USU Conference on Small Satellites, 2019

[20] KRAKsat dashboard, SatNOGS, [Online]. Available: https://dashboard.satnogs.org/d/AmlwwJvWz/kraksat?orgId=1\&refresh $=30$ s $\left(\right.$ accessed on $2^{\text {nd }}$ August 2021)

[21] PW-Sat2 Communication System and Ground Station. Crticial Design Review, PW-Sat2, Students' Space Association, Warsaw University of Technology, [Online]. Available: https://pw-sat.pl/wpcontent/uploads/2014/07/PW-Sat2-C-02.00-COMM-CDR.pdf (accessed on $25^{\text {th }}$ July 2021)

[22] K. Rankin, I. McNeil, I. Rankin, S. Stochaj, How not to build a CubeSat - Lessons Learned from Developing and Launching NMSU's First CubeSat, 33rd Annual AIAA/USU Conference on Small Satellites, 2019

[23] J. Praks et al., Aalto-1, multi-payload CubeSat: design, integration and launch, Acta Astronautica, 2021, submitted for publication

[24] L. Kepko et al., Dellingr: Reliability lessons learned from on-orbit, 32nd Annual AIAA/USU Conference on Small Satellites, 2018

[25] H. Leppinen et al., Developing a Linux-based nanosatellite on-board computer: Flight results from the Aalto-1 mission in IEEE Aerospace and Electronic Systems Magazine, 2019, DOI: 10.1109/MAES.2019.170217

[26] M. Doyle et al., Mission testing for improved reliability of CubeSats, International Conference on Space Optics, 2021

[27] A. G. Romero, L. Carlos Gadelha De Souza, Stability Evaluation of the SDRE Technique based on Java in a CubeSat Attitude and Orbit Control Subsystem, WSEAS Transactions on Systems, ISSN / E-ISSN: 1109-2777 / 2224-2678, Volume 20, 2021, Art. \#1, pp. 1-8
Creative Commons Attribution License 4.0 (Attribution 4.0 International, CC BY 4.0)

This article is published under the terms of the Creative Commons Attribution License 4.0 https://creativecommons.org/licenses/by/4.0/deed.en_US 